\title{
A Survey on Vision-based Dynamic Gesture Recognition
}

\author{
Sumpi Saikia \\ Dept. of Computer Science and Engineering \\ Tezpur University
}

\author{
Sarat Saharia \\ Dept. of Computer Scienceand Engineering \\ Tezpur University
}

\begin{abstract}
Gesture is the most primitive way of communication among human being. Today in the era of modern technology gesture recognition influences the world very diversely, from the physically challenged people to robot control to virtual reality environments. Compared to the systems which use extra devices (gloves, sensors), vision-based systems are more userfriendly and simple. Vision-based systems are easy to use, but most difficult to implement. This paper presents a comprehensive survey on the vision-based dynamic gesture recognition approaches, a comparative study on those methods, and find out the issues and challenges in this area.
\end{abstract}

\section{Keywords}

Gesture recognition, computer vision, dynamic gesture recognition, full body gesture recognition.

\section{INTRODUCTION}

Gesture is some meaningful expression made by human body parts to be communicated to others. It is a non-verbal communication which is the only way for physically challenged especially hearing-impaired people. Gesture recognition is the process by which the expression or gesture made by the user are recognized by the receiver [1]. Today, computer and computer-based devices are influencing human's life in every step. Gesture, as a media in humancomputer interaction (HCI) makes the world more userfriendly and flexible. Moreover, applications of gesture recognition can be found in in diversed areas like medicine [2], education [3], sports [4], dance [5], [6], etc.

The approach of gesture recognition can be glove-based and vision-based. In glove-based approach of gesture recognition, the user requires to wear a device and carry a load of cables connecting the device to a computer. Though the vision-based approach devoid of these problems, it has many other challenges. For example, the complex and cluttered backgrounds, dynamic lighting conditions and a deformable human hand shape, wrong object extraction can cause a machine to misunderstand the gesture [7].

The gestures can be broadly categorized into the following groups which are shown in Table 1 [1].

- Hand and arm gestures

- Head and face gestures

- $\quad$ Body gestures

Table 1. Types of gestures [1]

\begin{tabular}{|l|l|}
\hline \multicolumn{1}{|c|}{ Gesture type } & \multicolumn{1}{|c|}{ Example } \\
\hline Hand and arm gestures & $\begin{array}{l}\text { Recognition of hand poses, sign } \\
\text { languages, and entertainment } \\
\text { applications. }\end{array}$ \\
\hline $\begin{array}{l}\text { Head and face } \\
\text { gestures }\end{array}$ & $\begin{array}{l}\text { Nodding or shaking of head, } \\
\text { direction of eye gaze, raising } \\
\text { eyebrows, opening mouth to speak, }\end{array}$ \\
\hline
\end{tabular}

\begin{tabular}{|l|l|}
\hline \multirow{3}{*}{ Body gestures } & $\begin{array}{l}\text { winking, flaring the nostrils, looks } \\
\text { of surprise, anger, sadness, etc. }\end{array}$ \\
\hline & $\begin{array}{l}\text { Analyzing movements of a dancer } \\
\text { for generating matching music and } \\
\text { graphics, recognizing human gaits } \\
\text { for medical rehabilitation and } \\
\text { athletic training. }\end{array}$ \\
\hline
\end{tabular}

Gestures can be of static (posture) or dynamic (gesture) [8] Posture is a static command (e.g., stop signal) and the command is still for a short span of time. Posture is simple and needs less computation [9] as compared to gestures which are complex and for that it is suitable for real time environments [9], [1].

Gesture can be thought of as a sequence of postures. Gestures are dynamic over a period of time, i.e. it considers the expressions which are in motion (e.g., waving good bye). Some gestures include combination of static and dynamic characteristics as in sign languages.

\subsection{Motivation}

Recognition of dynamic gestures using vision-based approaches is a very challenging task, but this approach is more convenient to user. The area of vision-based gesture recognition leads to a large body of literature describing different body parts for gesture recognition including static and dynamic gestures. The purpose of this survey is to analyse some important surveys and articles on dynamic gesture recognition of hand and body gestures and pros and cons of those methods.

\subsection{Organization}

The remainder of this paper is organized as follows. Section 2 describes the approaches of gesture recognition. In section 3, various methods of vision based dynamic gesture recognition used by different researchers are discussed and their pros and cons are analyzed. Section 4 discusses the challenges in this area. The applications of dynamic gesture recognition are discussed in section 5. Finally the conclusion is given in section 6 .

\section{APPROACHES OF GESTURE RECOGNITION}

The approaches of gesture recognition can be broadly categorized into two groups:

\subsection{Glove-based Approaches}

In this approach, the user needs to wear a glove which employs mechanical or optical sensors attached to it that transforms finger or flexions into electrical signals to determine the hand posture [11]. A body suit is required to wear for body gesture recognition. The user needs to carry a load of cables that connect the device to a computer that hinders the ease and naturalness of the user interaction in computer controlled environment. Some of the research works in this approach can be found in [12], [13], [14], [15]. 


\subsection{Vision-based Approaches}

Vision-based approaches are more user-friendly and do not require any extra devices for analyzing gestures. It is the most natural way of user interaction as human perceives information from their surrounding. The methods under this approach deal with some properties such as texture and color [1] for analyzing gesture; while tracking devices cannot. In this method the input images or videos are captured by using camera(s) [16]. Although these methods are simple but a lot of challenges are involved such as the complex background, lighting variation, noisy videos, besides system requirements such as recognition time, robustness, and computational efficiency [17], [18].

\section{VISION-BASED DYNAMIC GESTURE RECOGNITION}

A dynamic gesture can be constituted by three distinct motion phases [10]: preparation, stroke and retraction. The stroke is the salient gestural movement. The preparation phase orients the body parts for the stroke and the retraction phase returns it to rest or orients for the next gestural stroke. The stroke is differentiable from the other two phases in velocity and acceleration.

Here different approaches of dynamic hand gesture recognition and full body gesture recognition are discussed.

\subsection{Hand and Arm Gesture Recognition}

Vision based hand gesture recognition approaches can be grouped into: 3D Model Based approaches and Appearance Based approaches.

\subsubsection{D Model based Approaches}

Model based approaches use some hand parameters like, palm pose, joint angles from the input image, and make 2D projection from 3D hand models [20]. Model-based approaches [21], [22], [18] estimate the current hand state by matching a 3D hand model to the observed image features. The difficulty of this approach is in feature extraction because human hands are texture less and do not provide reliable edges internally [23]. 3D Models can be classified into volumetric and skeletal models [7]. Volumetric models deal with 3D visual appearance of human hand and usually used in real time applications [24], [25]. The main problem with this modeling technique is that it deals with all the parameters of hand which are of huge dimensionality. Skeletal models overcome this problem of volumetric hand parameters models by limiting the set of parameters in modeling hand shapes from 3D structures [22].

\subsubsection{Appearance based Approaches}

In these approaches which are also known as View-Based Approaches, hand image is reconstructed using features extracted from visual appearance of the input image. It models the hand using the intensity of 2D images and compares these modeled features with features extracted from input camera(s) or video input [20]. The difficulties of 3D model based approaches can be overcome by using appearance based approaches [23].

Table 2 summarizes the features extracted, tracking methods, recognition methods and their limitations in their application areas.

A method for online gesture recognition systems is described in [26]. It is an HMM-based method that performed well for isolated gesture but not for continuous gesture recognition. It cannot segment the gestures automatically.
Hand gesture recognition has a great impact in the domain of robot control. In [25], they have presented a fast and simple algorithm for hand gesture recognition for robot control application. The algorithm is invariant to translation, rotation and scale of the hand. But they have considered a limited set of gestures. In [27], their recognition strategy is a combination of static shape recognition using Contour Discriminant Analysis, Kalman filter based tracking and HMM-based temporal characterization scheme. The tracking rate is about 20 fps (frame per second) and the complete system works at 25 fps. This method can automatically segment the start and end of continuous gesture. But it cannot handle the continuous deformation of hand shapes. [28] describes a method of guiding a robot using hand gesture from a distance, based on 2D skeleton representation of the hand and orientation histogram. Local orientation histogram is computed for static hand gesture recognition and dynamic signature is computed for each gesture for dynamic gesture recognition. Conventional template matching method is used for classification. This method is suitable for limited set of alphabet under uniform background.

A large body of literature is carried out in the domain of sign language recognition where hand gesture recognition is very effective. [31] presents a dynamic hand gesture recognition method that uses Dynamic Bayesian Network (DBN) model. This method works well for isolated gestures compared to continuous gestures. [32] recognizes Taiwan sign language using product HMM for recognition. In this method there is no appropriate fixed threshold to distinguish non-sign segments. In [36], they propose a system for recognizing 3D dynamic signs of Indian Sign Language (ISL). They have used Kinect camera for capturing 3D dynamic gestures of ISL words. In this method, features are extracted from the signs and converted to the intended textual form. This method integrates both local as well as global information of the dynamic sign. Using the concept of Axis of Least Inertia (ALI), a new trajectory based feature extraction method has been developed. The result has shown that performance of the system has been improved as integrating the local features with the global. A work on sign language recognition is presented in [38] that use Dynamic Time Wrapping for gesture recognition. The system has a limitation that it is capable of detecting the gestures which doesn't involve specific finger movements. [39] proposed a continuous hand gesture recognition algorithm which simultaneously divide the continuous gestures into disjointed gestures and recognize them. They have not considered any restricted assumptions for the motion of the hand between the disjointed gestures. Determining the borders for disjointed gestures in continuous gesture recognition is a challenging task. This work can easily perform the task. Segmentation and recognition of continuous gestures are done simultaneously. Their experimental results showed a recognition rate of $91.3 \%$ for disjointed gestures and $90.4 \%$ for continuous gestures. In this method, error sources can be viewing angle of camera, lighting condition, different clothing for users, etc.

A lot of work has been carried out for dynamic hand gesture recognition mainly for $\mathrm{HCI}$ applications. An innovative method of dynamic gesture recognition is to convert the recognition of dynamic gestures to the fusion of static frame images [37]. This method comprises of two steps: Firstly, they combine frame fusion with density distribution features for rough gesture recognition and secondly second, the hausdorff distance or fingertip detection is used for accurate gesture recognition. The recognition rate is above $90 \%$ from their experiment. [35] proposes a robust system for dynamic 
gesture recognition. In the first step hand is detected in every image frame obtained from USB camera, through skin segmentation and hand feature extraction. $\mathrm{YCrCb}$ color space is used for hand region segmentation and detected by detecting the number of fingers. Then, for hand tracking the algorithm based on ellipse fitting and motion feature is used. Finally, they have developed an algorithm for trajectory recognition. The dynamic gesture recognition method proposed by [33] can handle gestures of four types: graphic gesture, figure gesture, character gesture and action gesture. This is a HMM-based method to recognize complex singlehanded gesture. Adding the new features, hand size and hand shape, more complex gestures can be recognized. The proposed state-based spotting algorithm can divide the continuous gestures effectively. The total recognition rate is 96.67\%. Another HMM-based automatic dynamic gesture recognition system is proposed by [19]. In this method, the user's hand is detected using Adaboost algorithm with (Histograms of Gradient) HOG features. After hand detection the hand color model is updated which will be used in hand tracking. They have implemented a contour-based hand tracker. Features are extracted after the trajectory is obtained from the tracking algorithm. These features are used to compute the probability of each gesture type with HMM.

Vision-based dynamic hand gesture recognition is still a challenging task and there is scope in various application domains.

\subsection{Full Body Gesture Recognition}

Full body gesture recognition has a wide range of applications in sports, robotics, patient monitoring, dance etc. Since human body is highly articulated in nature, it is very difficult to recognize full body gestures from video. From the literature survey it is found that works on full body gesture recognition are comparatively less than hand gesture recognition.
Table 3 summarizes some methods of full body gesture recognition along with their particular application.

A view-invariant gesture recognition framework using voxel data obtained through visual hull reconstruction from multiple cameras is presented in [40]. View-invariant pose descriptors are extracted using multilinear analysis. Gestures are then treated as sequences of pose descriptors and HMM are used for gesture recognition. The recognition rate is good for isolated gestures.

In [69] a view-invariant video-based full body gesture recognition system is proposed. Multilinear analysis is performed on the silhouette images of the static poses making up the gestures by tensor decomposition and projection. The pose vectors are the inputs to the HMM for gesture recognition. This is the first system that addresses full body human gesture recognition from video without the recovery of body kinematics or $3 \mathrm{D}$ volumetric reconstruction.

A new effective and efficient feature extraction method is presented in [41] for online human gesture recognition. They solve the problem of how to continuously recognize gestures from unsegmented streams and how to differentiate different styles of a same gesture from other types of gestures. This method is applicable in interactive systems.

Full body gesture recognition has application in the field of dance. It is used for dance gesture recognition, performance evaluation of a dance,

In [70], an approach for dance gesture performance evaluation of Bali traditional dance is presented. This method can be used as alternative of dance gesture recognition.

From the literature survey it is found that full body dynamic gesture recognition has wide range of applications and there is scope to work in this area. 
Table 2: Different methods of vision-based hand gesture recognition

\begin{tabular}{|c|c|c|c|c|c|c|}
\hline Author & Year & $\begin{array}{l}\text { Continuous } \\
\text { /Isolate } \\
\text { Gesture }\end{array}$ & Features & $\begin{array}{l}\text { Recognition } \\
\text { /Classification } \\
\text { Method }\end{array}$ & Application & Disadvantage \\
\hline [26] & 1998 & Isolated & $\begin{array}{l}\text { Centre of motion, mean } \\
\text { absolute deviation from } \\
\text { centre of motion, } \\
\text { intensity of motion }\end{array}$ & HMM & $\begin{array}{l}\text { Online gesture } \\
\text { recognition systems }\end{array}$ & $\begin{array}{l}\text { Works well only for } \\
\text { continuous recognition of } \\
\text { isolated gestures. }\end{array}$ \\
\hline [27] & 2003 & Continuous & $\begin{array}{l}\text { Hand shape, motion } \\
\text { pattern }\end{array}$ & HMM & $\begin{array}{l}\text { Remote robot } \\
\text { control }\end{array}$ & $\begin{array}{l}\text { Continuous deformation of } \\
\text { hand shapes is not } \\
\text { permitted. }\end{array}$ \\
\hline$[28]$ & 2005 & Continuous & $\begin{array}{l}\text { Distance, velocity and } \\
\text { acceleration } \\
\text { information, energy } \\
\text { measurements, or angle } \\
\text { information }\end{array}$ & $\begin{array}{l}\text { Conventional } \\
\text { template } \\
\text { matching }\end{array}$ & Robot control & $\begin{array}{l}\text { Gives satisfactory } \\
\text { performance only for limited } \\
\text { alphabet under uniform } \\
\text { background }\end{array}$ \\
\hline$[25]$ & 2006 & Continuous & $\begin{array}{l}\text { 1D binary signal based } \\
\text { on centre of gravity }\end{array}$ & $\begin{array}{l}\text { Using their own } \\
\text { algorithm }\end{array}$ & Robot control & $\begin{array}{l}\text { It considers a limited } \\
\text { number of gestures. }\end{array}$ \\
\hline [29] & 2009 & Continuous & Edge, fingertip & $\begin{array}{l}\text { FSM and Fuzzy } \\
\text { c-mean } \\
\text { algorithm }\end{array}$ & HCI & $\begin{array}{l}\text { Works for a small set of } \\
\text { gestures. }\end{array}$ \\
\hline [30] & 2010 & Continuous & $\begin{array}{l}\text { Moment invariant } \\
\text { features }\end{array}$ & $\begin{array}{l}\text { Support Vector } \\
\text { Machine(SVM) }\end{array}$ & To simplify HCI & $\begin{array}{l}\text { The gestures that follow } \\
\text { 'start'gesture needs to be } \\
\text { made consciously. }\end{array}$ \\
\hline [31] & 2010 & Continuous & Hand motion & $\begin{array}{l}\text { Dynamic } \\
\text { Bayesian } \\
\text { Network }\end{array}$ & $\begin{array}{l}\text { Sign language } \\
\text { recognition }\end{array}$ & $\begin{array}{l}\text { Works well for isolated } \\
\text { gestures compared to } \\
\text { continuous gestures. }\end{array}$ \\
\hline [32] & 2011 & Continuous & $\begin{array}{l}\text { Hand movement, hand } \\
\text { shape, motion trajectory. }\end{array}$ & Product HMM & $\begin{array}{l}\text { Taiwan sign } \\
\text { language } \\
\text { recognition }\end{array}$ & $\begin{array}{l}\text { No appropriate fixed } \\
\text { threshold to distinguish non- } \\
\text { sign segments. }\end{array}$ \\
\hline [33] & 2012 & Continuous & $\begin{array}{l}\text { Hand position, velocity, } \\
\text { size and shape }\end{array}$ & HMM & Virtual reality & $\begin{array}{l}\text { This method can handle only } \\
\text { single-handed gestures. }\end{array}$ \\
\hline [19] & 2012 & Continuous & Orientation & HMM & $\mathrm{HCI}$ & $\begin{array}{l}\text { The tracking algorithm is } \\
\text { very sensitive to light. }\end{array}$ \\
\hline [34] & 2012 & Continuous & $\begin{array}{l}\text { Biggest contour, Convex } \\
\text { hull of the contour }\end{array}$ & Haar classifier & $\begin{array}{l}\text { Virtual games, } \\
\text { browsing images etc } \\
\text { in virtual } \\
\text { environment }\end{array}$ & $\begin{array}{l}\text { Less robust in recognition } \\
\text { phase. }\end{array}$ \\
\hline$[35]$ & 2013 & Isolated & $\begin{array}{l}\text { Hand shape, No. of } \\
\text { fingers }\end{array}$ & HMM & HCI & $\begin{array}{l}\text { The trajectory segmentation } \\
\text { algorithm can recognize } \\
\text { only some simple gestures. }\end{array}$ \\
\hline [36] & 2013 & Isolated & $\begin{array}{l}\text { Axis of least inertia, } \\
\text { distance from centroid of } \\
\text { the hand to fingertip }\end{array}$ & - & $\begin{array}{l}\text { Complex dynamic } \\
\text { ISL recognition }\end{array}$ & $\begin{array}{l}\text { The system is not complete, } \\
\text { as for complete recognition } \\
\text { of sign language, } \\
\text { information about head, } \\
\text { arm, facial expression are }\end{array}$ \\
\hline
\end{tabular}




\begin{tabular}{|c|c|c|c|c|c|l|}
\hline & & & & & & essential. \\
\hline$[37]$ & 2013 & Continuous & $\begin{array}{l}\text { Density distribution } \\
\text { feature }\end{array}$ & $\begin{array}{c}\text { Hausdorff } \\
\text { distance, } \\
\text { Fingertip } \\
\text { detection }\end{array}$ & $\begin{array}{l}\text { Human computer } \\
\text { interaction(HCI) }\end{array}$ & Time cost is not efficient. \\
\hline$[38]$ & 2014 & Continuous & Joint of interest & $\begin{array}{c}\text { Dynamic Time } \\
\text { Wrapping }\end{array}$ & $\begin{array}{c}\text { Sign language } \\
\text { recognition }\end{array}$ & $\begin{array}{l}\text { System is capable of } \\
\text { detecting the gestures which } \\
\text { doesn't involve specific } \\
\text { finger } \\
\text { movements. }\end{array}$ \\
\hline [39] & 2015 & Continuous & $\begin{array}{l}\text { SIFT (Scale Invariant } \\
\text { Feature Transform) } \\
\text { feature }\end{array}$ & $\begin{array}{l}\text { Template } \\
\text { matching }\end{array}$ & $\begin{array}{l}\text { ASL recognition } \\
\text { Error sources can be } \\
\text { viewing angle of camera, } \\
\text { lighting condition, different } \\
\text { clothing for users, etc. }\end{array}$ \\
\end{tabular}

Table 3: Different methods of vision-based full body gesture recognition

\begin{tabular}{|c|c|c|c|c|c|}
\hline Author & Year & Features & $\begin{array}{l}\text { Classification/ } \\
\text { Recognition } \\
\text { Method }\end{array}$ & Application & Description \\
\hline [69] & 2008 & $\begin{array}{c}\text { Pose vectors from 2D } \\
\text { silhouette }\end{array}$ & HMM & $\begin{array}{l}\text { Interactive environments } \\
\text { which use full body } \\
\text { gestures as control } \\
\text { signal. }\end{array}$ & $\begin{array}{l}\text { This system is } \\
\text { independent of view } \\
\text { angle of the camera. }\end{array}$ \\
\hline [40] & 2009 & 2D silhouette, Visual hull & HMM & $\begin{array}{l}\text { multi-person gesture } \\
\text { driven human computer } \\
\text { interactions }\end{array}$ & $\begin{array}{l}\text { Works for isolated } \\
\text { gesture. }\end{array}$ \\
\hline [70] & 2012 & Skeleton & $\begin{array}{l}\text { Finite State } \\
\text { Machine }\end{array}$ & $\begin{array}{l}\text { Performance evaluation } \\
\text { Bali traditional dance }\end{array}$ & $\begin{array}{l}\text { This method can be } \\
\text { used as alternative of } \\
\text { dance gesture } \\
\text { recognition }\end{array}$ \\
\hline$[41]$ & 2013 & Structured streaming skeletons & $\begin{array}{l}\text { Jointly sparse } \\
\text { coding }\end{array}$ & Interactive systems & $\begin{array}{l}\text { A new efficient } \\
\text { feature extraction } \\
\text { method is used }\end{array}$ \\
\hline [5] & 2014 & Skeleton of the body & HMM & $\begin{array}{l}\text { Kazakh dance gesture } \\
\text { recognition }\end{array}$ & $\begin{array}{l}\text { Recognition is based } \\
\text { on head movement }\end{array}$ \\
\hline
\end{tabular}

\section{CHALLENGES IN DYNAMIC GESTURE RECOGNITION}

The most promising challenges in dynamic gesture recognition systems [42] are discussed below:

- Scale problem: When the hand poses have different sizes in the gesture image, this problem arises [43].

- Translation problem: The variation of hand position in different images can also lead to erroneous representation of the features [44].
- Uncontrolled environment: In computer vision, the HCI systems can be performed in a controlled environment only. It is expected to work on unrestricted environment with wide range of lighting conditions which is still a challenging issue in computer vision [7].

- Rotation problem: When the hand region rotates in any direction this problem arises [43]. 
- Background problem: This problem arises when the back ground contains any skin-colored objects, which leads to misclassification [45].

- Variation of illumination conditions: Any change in the lighting conditions greatly affect in skin segmentation [45].

\section{APPLICATION AREAS}

Gesture recognition has a wide range of applications. It is used not only for hearing impaired people, but also it has applications in various fields. The various applications of gesture recognition are discussed below[46], [7], [47]:

- Sign language recognition: Sign language is one of the most natural and expressive ways for hearing impaired. A lot of systems have been developed on different sign languages to recognize gestures. An HMM- based real-time sign language recognition system is developed to interpret American Sign Language(ASL) [48], [49]. Japanese Sign Language (JSL) interpreter is proposed by [50] using Recurrent Neural Network. Using two different types of Neural Network, Partially and Fully Recurrent neural Network, an Arabic Sign Language(ArSL) recognition system is proposed [51]. Korean Sign Language (KSL) is recognized by [52]. [53] define a new gesture recognition algorithm for Korean scripts. A lexicon of 250 vocabularies in Taiwanese Sign Language (TWL) is recognized by [54].

- Robot control: To control robot using gestures is one of the interesting applications in this area [25], [24]. In [24], they proposed a system that uses the numbering to count the five fingers for controlling a robot using hand pose signs. For a robot manipulator pointing can be used to specify positions on a $2 \mathrm{D}$ workspace as guidance [55].

- Graphic editor control: Graphic editor control system requires the hand gesture to be tracked and located as a preprocessing operation. It uses 12 dynamic gestures for drawing and editing graphic system. Shapes for drawing are; triangle, rectangular, circle, arc, horizontal and vertical line for drawing, and commands for editing graphic system are; copy, delete, move, swap, undo, and close [7].

- Virtual environments: Gestures have considered as one of the effective means for virtual environments [23]. Virtual reality interaction uses the hand gesture to manipulate the virtual movements using one or two hands for 2D and 3D interactions display [23]. In [22], they provided 3D pointing gesture recognition for natural human computer Interaction $\mathrm{HCI}$ in a real-time from binocular views. Some virtual reality applications are available in [56], [57], [58].

- Games: A various game based applications have been developed using gesture recognition technology. Gesture recognition has applied on virtual game applications by [59]. In [60], they used hierarchical recognition of human gestures for sports video annotation. Using computer vision and gesture recognition techniques, [61] developed a vision based low cost input device for controlling the VLC player through gestures.

- Number recognition: An application of hand gesture is recognizing numbers. An automatic system that could isolate and recognize a meaningful gesture from hand motion of Arabic numbers from 0 to 9 in a real time system using HMM is found in [62].

- $\quad$ Surgical system: Hand gesture recognition systems can help doctors in surgical environment to manipulate digital images during medical procedures using hand gestures instead of touch screens or computer keyboards [7].

- Television control: Hand gestures have been used to develop systems to control a television set as to change the channel, turn the television on and off, increase and decrease the volume, and mute the sound [63].

- 3D modeling: For 3D modeling, to create 3D objects the shape of the hand should be determined by the user [64]. Various applications of 3D modeling can be found in [65], [66], [67], [68]. 3D hand modeling is still a promising field of research [64].

- Dance: Dance gesture recognition is used for performance evaluation of dance, e-learning of dance etc.

- Medicine: Medically monitoring patients' emotional states or stress levels.

- Education: Distance learning/ tele-teaching assistance

\section{CONCLUSION}

In today's world, people are demanding the evolution of touch to touchless technology. The Natural User Interfaces are simple and easy to use, but very difficult to implement. In this paper, vision-based approaches for dynamic gesture recognition techniques have been reviewed. This is a very challenging research area and comparatively a less work has been carried out then static gesture recognition till now. A satisfactory result can be found only under controlled environment. Recognition of dynamic gestures compared to static gestures needs more computation. More stress should be given to build up a dynamic gesture recognition system which gives satisfactory performance. From the comparative analysis of some papers on dynamic gesture recognition it is observed the scope towards this direction could be gesture segmentation or gesture spotting from video. Another direction could be to develop efficient algorithm for extraction of relevant features and feature selection that leads to good recognition. From the survey it is observed that there is lack of proper dataset of dynamic gestures which hinders to measure the effectiveness of the research compared to other's methods. So developing proper dataset could extend research in this area.

\section{REFERENCES}

[1] Mitra, S. and Acharya, T. 2007. Gesture recognition: A survey. Systems, Man, and Cybernetics, Part C: Applications and Reviews, IEEE Transactions on, 37(3), 311-324.

[2] Englmeier, K-H., et al. "Virtual reality and multimedia human-computer interaction in medicine." Multimedia 
Signal Processing, 1998 IEEE Second Workshop on. IEEE, 1998.

[3] Thakkar, Varun, et al. "Learning Math Using Gesture." Education and e-Learning Innovations (ICEELI), 2012 International Conference on. IEEE, 2012.

[4] Takano, Kosuke, and Kin Fun Li. "Classifying sports gesture using event-based matching in a multimedia elearning system." Advanced Information Networking and Applications Workshops (WAINA), 2012 26th International Conference on. IEEE, 2012.

[5] Nussipbekov, A. K., E. N. Amirgaliyev, and Minsoo Hahn. "Kazakh Traditional Dance Gesture Recognition." Journal of Physics: Conference Series. Vol. 495. No. 1. IOP Publishing, 2014.

[6] James, Jodi, et al. "Movement-based interactive dance performance."Proceedings of the 14th annual ACM international conference on Multimedia. ACM, 2006.

[7] Thomas, M. C., and Pradeepa, A. P. M. S. 2014. A COMPREHENSIVE REVIEW ON VISION BASED HAND GESTURE RECOGNITION TECHNOLOGY. International Journal, 2(1).

[8] Pavlovic, V., Sharma, R., and Huang, T. S. 1997. Visual interpretation of hand gestures for human-computer interaction: A review. Pattern Analysis and Machine Intelligence, IEEE Transactions on, 19(7), 677-695.

[9] Li, X. 2003. Gesture recognition based on fuzzy CMeans clustering algorithm.Department Of Computer Science The University Of Tennessee Knoxville.

[10] Quek, F. 1994. Toward a vision-based hand gesture interface. InVirtual Reality Software and Technology Conference (pp. 17-29).

[11] Moeslund, T. B., and Granum, E. 2001. A survey of computer vision-based human motion capture. Computer vision and image understanding, 81(3), 231-268.

[12] Baudel, T., and Beaudouin-Lafon, M. 1993. Charade: remote control of objects using free-hand gestures. Communications of the ACM, 36(7), 28-35.

[13] Fels, S. S., and Hinton, G. E. 1993. Glove-talk: A neural network interface between a data-glove and a speech synthesizer. Neural Networks, IEEE Transactions on, 4(1), 2-8.

[14] Sturman, D. J., and Zeltzer, D. 1994. A survey of glovebased input. Computer Graphics and Applications, IEEE, 14(1), 30-39.

[15] Quam, D. L. 1990. Gesture recognition with a dataglove. In Aerospace and Electronics Conference, 1990. NAECON 1990., Proceedings of the IEEE 1990 National (pp. 755-760). IEEE.

[16] Meena, S. 2011. A Study on Hand Gesture Recognition (Doctoral dissertation, National Institute of Technology, Rourkela)

[17] Stergiopoulou, E., and Papamarkos, N. 2009. Hand gesture recognition using a neural network shape fitting technique. Engineering Applications of Artificial Intelligence, 22(8), 1141-1158.

[18] Chaudhary, A., Raheja, J. L., and Raheja, S. 2012. A vision based geometrical method to find fingers positions in real time hand gesture recognition. Journal of Software, 7(4), 861-869.

[19] Wang, X., et al. "Hidden-markov-models-based dynamic hand gesture recognition." Mathematical Problems in Engineering 2012 (2012).

[20] Garg, P., Aggarwal, N., and Sofat, S. 2009. Vision based hand gesture recognition. World Academy of Science, Engineering and Technology, 49(1), 972-977.

[21] Pan, Z, et al. "A real-time multi-cue hand tracking algorithm based on computer vision." Virtual Reality Conference (VR), 2010 IEEE.

[22] Hackenberg, G., McCall, R., and Broll, W. 2011. Lightweight palm and finger tracking for real-time 3D gesture control. In Virtual Reality Conference (VR), 2011 IEEE (pp. 19-26). IEEE.

[23] Murthy, G. R. S., and Jadon, R. S. 2009. A review of vision based hand gestures recognition. International Journal of Information Technology and Knowledge Management, 2(2), 405-410.

[24] Ghobadi, S.E, et al. "Real time hand based robot control using multimodal images." IAENG International Journal of Computer Science 35.4 (2008): 500-505.

[25] Malima, A., Özgür, E., and Çetin, M. 2006. A fast algorithm for vision-based hand gesture recognition for robot control. In Signal Processing and Communications Applications, 2006 IEEE 14th (pp. 1-4). IEEE.

[26] Eickeler, S., Kosmala, A., and Rigoll, G. 1998. Hidden markov model based continuous online gesture recognition. In Pattern Recognition, 1998. Proceedings. Fourteenth International Conference on (Vol. 2, pp. 1206-1208). IEEE.

[27] Ramamoorthy, A., et al. "Recognition of dynamic hand gestures." Pattern Recognition 36.9 (2003): 2069-2081.

[28] Ionescu, B., et al. "Dynamic hand gesture recognition using the skeleton of the hand." EURASIP Journal on Applied Signal Processing 2005 (2005): 2101-2109.

[29] Verma, R., and Dev, A. 2009. Vision based hand gesture recognition using finite state machines and fuzzy logic. In Ultra Modern Telecommunications \& Workshops, 2009. ICUMT'09. International Conference on (pp. 1-6). IEEE.

[30] Zou, Z, et al. "Dynamic hand gesture recognition system using moment invariants." Information and Automation for Sustainability (ICIAFs), 2010 5th International Conference on. IEEE, 2010.

[31] Suk, H. I., Sin, B. K., and Lee, S. W. 2010. Hand gesture recognition based on dynamic Bayesian network framework. Pattern Recognition, 43(9), 3059-3072.

[32] Yu, S., et al. "Vision-based continuous sign language recognition using product HMM." Pattern Recognition (ACPR), 2011 First Asian Conference on. IEEE, 2011.

[33] Yang, Z., et al. "Dynamic hand gesture recognition using hidden Markov models." Computer Science \& Education (ICCSE), 2012 7th International Conference on. IEEE, 2012.

[34] Rautaray, S. S., and Agrawal, A. 2012. Real time hand gesture recognition system for dynamic applications. Int J UbiComp, 3(1), 21-31. 
[35] Jiang, X., et al. "A dynamic gesture recognition method based on computer vision." Image and Signal Processing (CISP), 2013 6th International Congress on. Vol. 2. IEEE, 2013.

[36] Geetha, M., et al. "A vision based dynamic gesture recognition of Indian Sign Language on Kinect based depth images." Emerging Trends in Communication, Control, Signal Processing \& Computing Applications (C2SPCA), 2013 International Conference on. IEEE, 2013.

[37] Zhang, T., and Feng, Z. 2013. Dynamic Gesture Recognition Based on Fusing Frame Images. In Intelligent Systems Design and Engineering Applications, 2013 Fourth International Conference on (pp. 280-283). IEEE.

[38] Masood, S., et al. "Dynamic time wrapping based gesture recognition."Robotics and Emerging Allied Technologies in Engineering (iCREATE), 2014 International Conference on. IEEE, 2014

[39] Nasri, S., Behrad, A., and Razzazi, F. 2015. A novel approach for dynamic hand gesture recognition using contour-based similarity images. International Journal of Computer Mathematics, 92(4), 662-685.

[40] Peng, B., Qian, G., and Rajko, S. 2009. View-invariant full-body gesture recognition via multilinear analysis of voxel data. In Distributed Smart Cameras, 2009. ICDSC 2009. Third ACM/IEEE International Conference on (pp. 1-8). IEEE

[41] Zhao, X., et al. "Online human gesture recognition from motion data streams."Proceedings of the 21st ACM international conference on Multimedia. ACM, 2013.

[42] Khan, R. Z., and Ibraheem, N. A. 2012. Comparative study of hand gesture recognition system. In Proc. of International Conference of Advanced Computer Science \& Information Technology in Computer Science \& Information Technology (CS \& IT) (Vol. 2, No. 3, pp. 203-213).

[43] Wysoski, S.G., et al. "A rotation invariant approach on static-gesture recognition using boundary histograms and neural networks." Neural Information Processing, 2002. ICONIP'02. Proceedings of the 9th International Conference on. Vol. 4. IEEE, 2002.

[44] Freeman, W. T., and Roth, M. 1995. Orientation histograms for hand gesture recognition. In International workshop on automatic face and gesture recognition (Vol. 12, pp. 296-301).

[45] Hasan, M. M., and Mishra, P. K. 2011. Performance Evaluation of Modified Segmentation on Multi Block For Gesture Recognition System. International Journal of Signal Processing, Image Processing and Pattern Recognition, 4(4), 17-28.

[46] Kaushik, D. M., and Jain, R. 2014. Gesture Based Interaction NUI: An Overview.arXiv preprint arXiv:1404.2364.

[47] Sarkar, A. R., Sanyal, G., and Majumder, S. 2013. Hand gesture recognition systems: a survey. International Journal of Computer Applications (0975-8887),71(15).

[48] Starner, T., and Pentland, A. 1997. Real-time american sign language recognition from video using hidden markov models. In Motion-Based Recognition (pp. 227 243). Springer Netherlands.

[49] Starner, T., Weaver, J., and Pentland, A. 1998. Real-time american sign language recognition using desk and wearable computer based video. Pattern Analysis and Machine Intelligence, IEEE Transactions on, 20(12), 1371-1375

[50] Murakami, K., and Taguchi, H. 1991. Gesture recognition using recurrent neural networks. In Proceedings of the SIGCHI conference on Human factors in computing systems (pp. 237-242). ACM.

[51] Maraqa, M., and Abu-Zaiter, R. 2008. Recognition of Arabic Sign Language (ArSL) using recurrent neural networks. In Applications of Digital Information and Web Technologies, 2008. ICADIWT 2008. First International Conference on the (pp. 478-481). IEEE.

[52] Kim, J. S., Jang, W., and Bien, Z. 1996. A dynamic gesture recognition system for the Korean sign language (KSL). Systems, Man, and Cybernetics, Part B: Cybernetics, IEEE Transactions on, 26(2), 354-359.

[53] Cho, M. G. 2006. A new gesture recognition algorithm and segmentation method of Korean scripts for gestureallowed ink editor. Information Sciences,176(9), 12901303.

[54] Liang, R. H., and Ouhyoung, M. 1998. A real-time continuous gesture recognition system for sign language. In Automatic Face and Gesture Recognition, 1998. Proceedings. Third IEEE International Conference on (pp. 558-567). IEEE.

[55] Cipolla, R., and Hollinghurst, N. J. 1996. Human-robot interface by pointing with uncalibrated stereo vision. Image and Vision Computing, 14(3), 171-178

[56] Bertsch, F., and Hafner, V. V. 2009. Real-time dynamic visual gesture recognition in human-robot interaction. In Humanoid Robots, 2009. Humanoids 2009. 9th IEEERAS International Conference on (pp. 447-453). IEEE.

[57] Portillo-Rodriguez, O., et al. "Development of a 3D real time gesture recognition methodology for virtual environment control." Robot and Human Interactive Communication, 2008. RO-MAN 2008. The 17th IEEE International Symposium on. IEEE, 2008.

[58] Guan, Y., and Zheng, M. 2008. Real-time 3D pointing gesture recognition for natural HCI. In Intelligent Control and Automation, 2008. WCICA 2008. 7th World Congress on (pp. 2433-2436). IEEE.

[59] Zhang, X., et al. "Hand gesture recognition and virtual game control based on $3 \mathrm{D}$ accelerometer and EMG sensors." Proceedings of the 14th international conference on Intelligent user interfaces. ACM, 2009.

[60] Chambers, G.S., et al. "Hierarchical recognition of intentional human gestures for sports video annotation." Pattern Recognition, 2002. Proceedings. 16th International Conference on. Vol. 2. IEEE, 2002.

[61] Rautaray, S. S., and Agrawal, A. 2010. A vision based hand gesture interface for controlling VLC media player. International Journal of Computer Applications, 10(7), 11-16.

[62] Elmezain, M., et al. "A hidden markov model-based isolated and meaningful hand gesture 
recognition." International Journal of Electrical, Computer, and Systems Engineering 3.3 (2009): 156163.

[63] Freeman, W. T., and Weissman, C. 1995. Television control by hand gestures. In Proc. of Intl. Workshop on Automatic Face and Gesture Recognition (pp. 179-183).

[64] LaViola, J. 1999. A survey of hand posture and gesture recognition techniques and technology. Brown University, Providence, RI.

[65] Krueger, M. W. 1991. Artificial reality II. AddisonWesley Professional.

[66] Weimer, D., and Ganapathy, S. K. 1992. Interaction techniques using hand tracking and speech recognition. In Multimedia interface design (pp. 109-126). ACM.
[67] Smith, G., et al. "3D scene manipulation with 2D devices and constraints." Graphics Interface. Vol. 1. 2001.

[68] Mapes, D. P., and Moshell, J. M. 1995. A two-handed interface for object manipulation in virtual environments. Presence: Teleoperators and Virtual Environments, 4(4), 403-416.

[69] Peng, B., Qian, G., and Rajko, S. 2008. View-invariant full-body gesture recognition from video. In Pattern Recognition, 2008. ICPR 2008. 19th International Conference on (pp. 1-5). IEEE.

[70] Heryadi, Y., et al. "A syntactical modelling and classification for performance evaluation of Bali traditional dance." ICACSIS 2012 